\title{
Association of High Serum Levels of Growth Factors with Good Outcome in Ischemic Stroke: a Multicenter Study
}

\author{
Tomás Sobrino ${ }^{1} \cdot$ Manuel Rodríguez-Yáñez ${ }^{1} \cdot$ Francisco Campos $^{1} \cdot$ Ramón Iglesias-Rey $^{1}$ (D) Mónica Millán ${ }^{2}$. \\ Natalia Pérez de la Ossa ${ }^{2}$. Antonio Dávalos ${ }^{2}$. Raquel Delgado-Mederos ${ }^{3}$ - Alejandro Martínez-Domeño ${ }^{3}$. \\ Joan Martí-Fábregas ${ }^{3} \cdot$ Mar Castellanos $^{4,5}$ - Joaquín Serena ${ }^{4}$ - Aida Lago ${ }^{6} \cdot$ Exuperio Díez-Tejedor $^{7}$. José Castillo ${ }^{1}$
}

Received: 1 August 2019 / Revised: 15 October 2019 / Accepted: 16 October 2019 / Published online: 25 November 2019

(C) The Author(s) 2019

\begin{abstract}
The main objective of this research work was to study the association of serum levels of growth factors (GF) and SDF-1 $\alpha$ with the functional outcome and reduction of lesion volume in ischemic stroke patients. In this multicenter study, 552 patients with nonlacunar stroke (male, 62.1\%; mean age, $68.2 \pm 11.4$ ) were included within $24 \mathrm{~h}$ from symptom onset. The main outcome variable was good functional outcome (modified Rankin Scale $[\mathrm{mRS}] \leq 2$ ) at 12 months. Secondary outcome variable was infarct volume (in $\mathrm{mL}$ ) after $6 \pm 3$ months. Serum levels of VEGF, Ang-1, G-CSF, BDNF, and SDF- $1 \alpha$ were measured by ELISA at admission, 7 \pm 1 days, at $3 \pm 1$ months, and $12 \pm 3$ months. Except for BDNF, all GF and SDF- $1 \alpha$ serum levels showed a peak value at day 7 and remained elevated during the first 3 months (all $p<0.01$ ). High serum levels at day 7 of VEGF (OR, 19.3), Ang-1 (OR, 14.7), G-CSF (OR, 9.6), and SDF-1 $\alpha(\mathrm{OR}, 28.5)$ were independently associated with good outcome at 12 months (all $p<0.0001)$. On the other hand, serum levels of VEGF (B, - 21.4), G-CSF (B, - 14.0), Ang-1 (B, - 13.3), and SDF-1 $\alpha$ (B, - 44.6) measured at day 7 were independently associated with lesion volume at 6 months $(p<0.01)$. In summary, high serum levels of VEGF, Ang-1, G-CSF, and SDF- $1 \alpha$ at day 7 and 3 months after ischemic stroke are associated with good functional outcome and smaller residual lesion at 1 year of follow-up.
\end{abstract}

Keywords Growth factors $\cdot$ Infarct volume $\cdot$ Ischemic stroke $\cdot$ Prognosis

\section{Introduction}

Improvement of the management of stroke patients, especially in developed countries, has contributed to the reduction in mortality and morbidity rates observed in recent decades. However, the incidence of stroke has not followed this same trend showing a continuous increase in developed countries

Tomás Sobrino

tomas.sobrino.moreiras@sergas.es

José Castillo

Jose.Castillo.Sanchez@sergas.es

1 Clinical Neurosciences Research Laboratory, Health Research Institute of Santiago de Compostela (IDIS), Santiago de Compostela, Spain

2 Department of Neurosciences - Acute Stroke Unit, Hospital Universitari Germans Trias i Pujol, Universidad Autònoma de Barcelona, Badalona, Spain
[1]. Besides, the demographic change expected in Europe for the next 50 years suggests that this situation will become worse [2].

In addition, the ceiling of reperfusion treatment is already close (only $20 \%$ of with ischemic stroke patients can benefit from these treatments in highly specialized hospitals, whose and their effectiveness does not exceed 50-60\%) [3].

3 Stroke Unit, Neurology Department, Hospital de la Santa Creu i Sant Pau, Barcelona, Spain

4 Department of Neurology - Stroke Unit, Biomedical Research Institute of Girona, Hospital Universitario Doctor Josep Trueta, Girona, Spain

5 Department of Neurology, Complexo Hospitalario Universitario da Coruña, A Coruña, Spain

6 Department of Neurology, Hospital Universitario La Fe, Valencia, Spain

7 Department of Neurology and Stroke Center, Neurosciences Area, IdiPAZ (Health Research Institute), La Paz University Hospital, Autónoma University of Madrid, Madrid, Spain 
Moreover, new neuroprotective approaches are limited, mainly due to a narrow therapeutic window. Therefore, the identification of new targets for ischemic stroke is mandatory for clinicians and researchers.

The medium-long-term prognosis (weeks and/or months) of patients with ischemic stroke will depend on the size and topography of the infarction, the speed and efficiency of the acute phase therapeutic measures, and the degree of neurorepair processes mediated by the patient's cerebral plasticity mechanisms $[4,5]$.

One of the main options for enhancing the mechanisms of cellular plasticity is cellular therapy that, favors by the extraordinary advance of the investigation of cellular biology, represents a hopeful therapeutic target for ischemic stroke. In this regard, the use of embryonic stem cells, induced pluripotent stem cells, and adult stem cells have demonstrated therapeutic effects in preclinical models of cerebral ischemia, and to a lesser extent in humans mainly through the mesenchymal stem cells. However, the mechanisms underlying cellular therapy are not well understood, since most of the transplanted cells disappear within a few weeks, and this therapy is not free from potentially serious complications [6-8]. It is possible that effectiveness of cellular therapy is based on trophic factors and other bioactive substances that modify the cerebral microenvironment and stimulate the development of the patient's own cellular niches [9-11]. Therefore, the stimulation of cerebral plasticity by using growth factors $(\mathrm{GF})$, which participate in a coadjutant way in neurogenesis, neoangiogenesis, gliagenesis, and synaptogenesis mechanisms, may constitute a more translational therapeutic approach from the lab to the bedside [12,13].

Unlike the great existing knowledge on GF in preclinical models of cerebral ischemia, there is a gap in studies in clinical practice which may be one of the reasons why clinical trials with GF such as granulocyte colony-stimulating factor (GCSF) have failed $[13,14]$. Thus, it is necessary to know better the relationship between growth factors and the prognosis of ischemic stroke patients at long term.

For this purpose, we designed a multicenter and observational study of a cohort of patients with acute ischemic stroke in order to study the association of serum levels of GF (vascular endothelial growth factor [VEGF], [G-CSF], brainderived neurotrophic factor $[\mathrm{BDNF}]$, angiopoietin 1 [Ang1]) and stromal-derived factor- $1 \alpha[\mathrm{SDF}-1 \alpha]$ with the functional outcome and reduction of lesion volume of ischemic stroke patients during a follow-up period of 1 year.

\section{Materials and Methods}

\section{Study Population and Patient Characteristics}

We have conducted a multicenter and observational study of a cohort of ischemic stroke patients, of less than $24 \mathrm{~h}$ from stroke onset, consecutively admitted to Stroke Units of 6 Spanish hospitals. The period of recruitment of the patients was carried out in the first half of 2014. Patients without diagnostic confirmation by neuroimaging, lacunar infarcts, previous disability (modified Rankin Scale $>1$ ), cancer, severe systemic or metabolic disease, and inflammatory or infectious disease in the previous 15 days and patients who received investigational drugs in clinical trials were excluded.

\section{Clinical Variables}

All patients were admitted to an acute Stroke Unit and treated according to the European Stroke Organisation guidelines [15]. The follow-up period was 1 year, including 2 visits at 3 \pm 1 months and at $12 \pm 3$ months.

Medical history recording potential vascular risk factors, blood and coagulation test, 12-lead electrocardiogram, chest $\mathrm{X}$-ray, and carotid ultrasonography were performed at admission. Stroke subtype was classified according to the TOAST criteria [16], and stroke severity was assessed by a certified neurologist using the National Institute of Health Stroke Scale (NIHSS) at admission, $48 \mathrm{~h}, 3 \pm 1$, and $12 \pm 3$ months. Functional outcome was evaluated at $3 \pm 1$ and $12 \pm 3$ months using the modified Rankin Scale (mRS). Good functional outcome was defined as a mRS score $\leq 2$. Physiotherapy/ rehabilitation was collected during the hospitalization as well as the first 3 or 12 months of follow-up period.

\section{Neuroimaging Variables}

Cerebral computed tomography (CT) studies were carried out at admission, between 4th and 7th days and after 3 months. Patients who received reperfusion treatment had another CT study before and at $24-36 \mathrm{~h}$ after treatment. Hemorrhagic transformation (HT) was assessed in the follow-up CT. HT was defined as symptomatic when it was associated with early neurological deterioration (worsening $>4$ points in the NIHSS during the first $48 \mathrm{~h}$ from stroke onset). Lesion volume was calculated on the follow-up CT by using the formula $0.5 \times \mathrm{A} \times$ $\mathrm{B} \times \mathrm{C}$, where $\mathrm{A}$ and $\mathrm{B}$ are the largest perpendicular diameters, and $\mathrm{C}$ is the number of $1-\mathrm{cm}$ thick sections that contain the lesion. All neuroimaging evaluations were made by investigators who had no knowledge of the patients' clinical and laboratory results.

\section{Outcome Variables}

The primary endpoint was good functional outcome (mRS score $\leq 2$ ) at $12 \pm 3$ months; as secondary variables, we considered the good functional outcome ( $\mathrm{mRS}$ score $\leq 2)$ at $3 \pm 1$ months and the lesion volumes between 4 th and 7 th days and after 3 months. 


\section{Laboratory Tests}

Biochemistry, hematology, and coagulation test were assessed in the central laboratory of each participating hospital. However, the growth factors and SDF- $1 \alpha$ determinations of the total patients included in each of 6 hospitals were performed in the Clinical University Hospital of Santiago de Compostela. The GF selected for this study were VEGF, GCSF, BDNF, Ang-1, and SDF- $1 \alpha$. For these molecular determinations, venous blood samples were collected in Vacutainer tubes (Becton Dickinson, San Jose, CA, USA) at admission, 7 \pm 1 days, $3 \pm 1$, and $12 \pm 3$ months. After allowing to clot for $60 \mathrm{~min}$, blood samples were centrifuged at $3000 \times \mathrm{g}$ for $10 \mathrm{~min}$, and the serum was immediately aliquoted, frozen, and stored at $-80^{\circ} \mathrm{C}$ until analysis. Serum levels of VEGF, G-CSF, and BDNF (Boster Biological Technology, Encyclopedia Circle Fremont, CA, USA), Ang-1 (Adipo Bioscience Inc., Santa Clara, CA, USA), and SDF-1 $\alpha$ (R\&D Systems Inc., Minneapolis, MN, USA) were quantified using commercial ELISA kits following instructions provided by commercial houses. The coefficients of intra-assay variation were VEGF, 4.4\%; G-CSF, 5.2\%; BDNF, 4.8\%; Ang-1, 4.7\%; and SDF$1 \alpha, 3.6 \%$; the coefficients of inter-assay variation were VEGF, 6.9\%; G-CSF, 8.3\%; BDNF, 7.9\%; Ang-1, 8.1\%; and SDF$1 \alpha, 7.2 \%$. The determinations were performed in an independent laboratory that did not have access to clinical or neuroimaging data.

\section{Statistical Analysis}

According to unpublished own previous results, $42 \%$ ( $\mathrm{p} 1=$ 0.42) of ischemic stroke patients have a mRS $>2$ at discharge and do not show a significant improvement during a 1 year of follow-up period. It has been calculated that if a sample of 545 individuals $(n=545)$ is included, an accuracy of $4.12 \%(\omega=$ 0.41 ) will be obtained by means of a normal $95 \%$ asymptotic confidence interval $(\gamma=0.95)$ bilateral $(c=2)$. For the calculation of the sample size, EPIDAT version 4.2 (www.sergas. es/Saude-publica/EPIDAT) was used.

Results were expressed as percentages for categorical variables and as mean (SD) or median and interquartile range for the continuous variables depending on their distribution. Kolmogorov-Smirnov test was used for testing normality. Chi-square or Fisher test was performed to study categorical variables. The continuous were studied with the Student's $t$ or the Mann-Whitney tests. Spearman's or Pearson's analyses were used to test bivariate correlations. ANOVA was used to analyze the relationship between stroke subtypes and growth factors. Mixed analysis of variance (MANOVA) was used to study the effect of time, and groups (good and poor outcome) by time (baseline to 12 months) interactions on GF and SDF$1 \alpha$ levels. The influence of growth factors on functional outcome and the lesion volume was assessed by logistic regression and multiple lineal regression models, respectively. The models were constructed considering the baseline variables related to each outcome variable. Results were expressed as adjusted odds ratios (OR) or Beta estimate with their 95\% confidence intervals $(95 \% \mathrm{CI})$. The statistical analysis was conducted using IBM SPSS Statistic, version 20 (SPSS Inc. Chicago, IL, USA).

\section{Results}

\section{Sample Description}

A total of 650 patients with a first-ever ischemic stroke of less than $24 \mathrm{~h}$ from symptoms onset was included in the study. Six patients were excluded due to lack of diagnostic confirmation, 39 patients were lacunar infarcts, 4 patients showed previous disability (mRS $>1$ ), and 12 patients were included in clinical trials during the acute phase. Moreover, 37 patients were loss during follow-up or suffered a recurrent stroke. Therefore, a final sample of 552 patients (male, 62.1\%; mean age, $68.2 \pm 11.4$ years) was valid for testing the primary endpoint of the study.

On the other hand, 28 patients died during the first month/ week/admission, 37 at 3 months, and 84 at the end of the follow-up period of 12 months. Additionally, blood samples were not obtained in 8 patients at 3 months, and 23 patients had not blood sample at 1 year of follow-up. So, a baseline serum sample was available in 552 patients, at 7 days in 524,

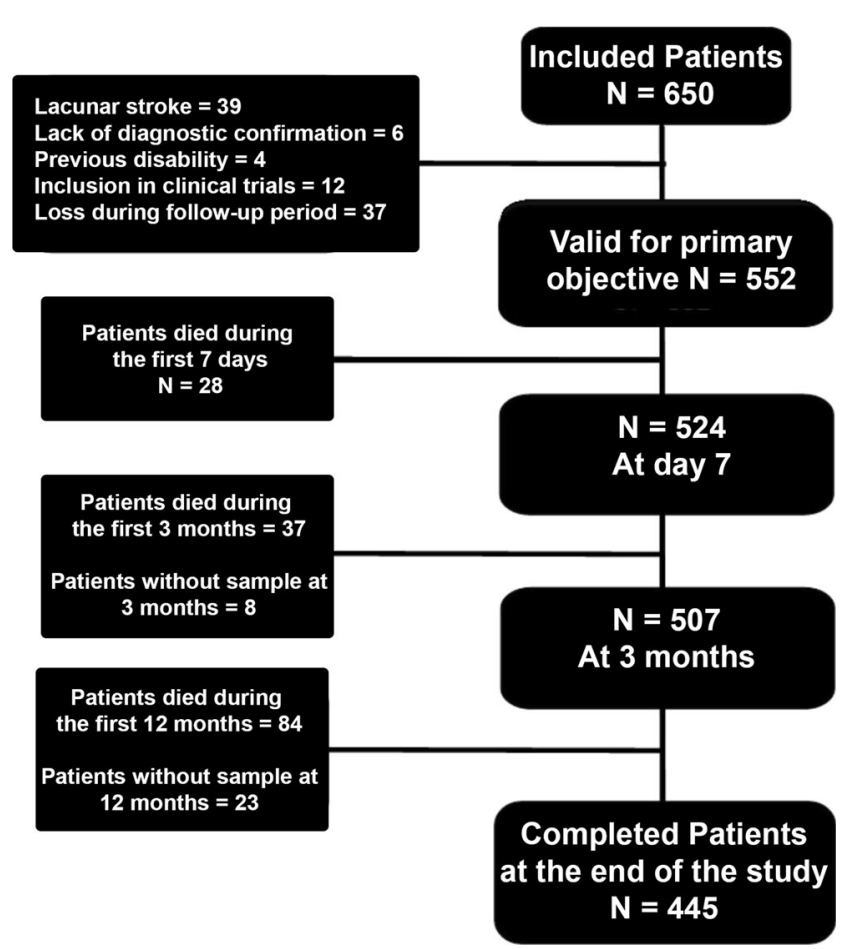

Fig. 1 Screening and enrollment into the study 
at 3 months in 507, and finally 445 patients had serum sample at 12 months (Fig. 1).

Regarding neuroimaging, CT at admission was performed in all; the second CT performed between 4th and 7th days was available in 537 patients, while the final CT was completed in 409 patients after 3 months (average time, $6.3 \pm 2.7$ months after admission).

NIHSS at admission was $11[6,17]$. A total of 198 patients $(35.9 \%)$ received intravenous fibrinolytic treatment within the first $4.5 \mathrm{~h}$. Following TOATS criteria, $13.9 \%$ of patients were diagnosed as atherothrombotic stroke, $50.0 \%$ cardioembolic, and $36.1 \%$ as undetermined origin. The median (quartiles) of mRS at 3 months was $2[1,3]$ and $1[0,3]$ at 12 months.

\section{Temporal Profile of GF and SDF-1a Levels}

Except for BDNF, all GF and SDF- $1 \alpha$ serum levels showed a peak value at day 7 (all $p<0.01$ ) (Fig. 2). The increase in percentage of serum levels of GF during the first week were as follows: VEGF, $77.4 \pm 111.8 \%(p<0.0001)$; G-CSF, $67.2 \pm$ 98.8\% ( $p<0.0001)$; BDNF, $-2.1 \pm 44.3 \%$ ( $p=0.349)$; Ang$1,69.6 \pm 120.6 \%(p<0.0001)$; and SDF- $1 \alpha, 29.6 \pm 31.4 \%(p$ $<0.0001)$. At 3 months, only an increase of VEGF $(p<$ $0.0001)$ and G-CSF $(p<0.0001)$ serum levels in relation to the basal concentration persisted; the concentrations of BDNF ( $p=0.312)$, Ang-1 ( $p=0.632)$, and SDF-1 $\alpha(p=0.078)$ were similar to baseline. By contrast, at 12 months, serum levels of VEGF $(p=0.169)$ and G-CSF $(p=0.418)$ were similar to those at baseline, but BDNF $(p<0.0001)$, Ang-1 ( $p=$ $0.016)$, and SDF- $1 \alpha(p<0.0001)$ levels were lower.

\section{Primary Outcome: Good Functional Outcome at 12 Months}

Table 1 shows the main characteristics of patients by functional outcome groups. Three hundred and fifty-one $(68.6 \%)$ patients
Fig. 2 (a-e) Temporal profile of GF (VEGF, G-CSF, BDNF, Ang1) and SDF- $1 \alpha$ levels at admission, $7 \pm 1$ days, $3 \pm 1$ months, and $12 \pm 3$ months. All GF and SDF- $1 \alpha$ serum levels showed a peak value at day 7 and remained elevated during the first 3 months (all $p<0.01$ ), except for $\mathrm{BDNF}$ a

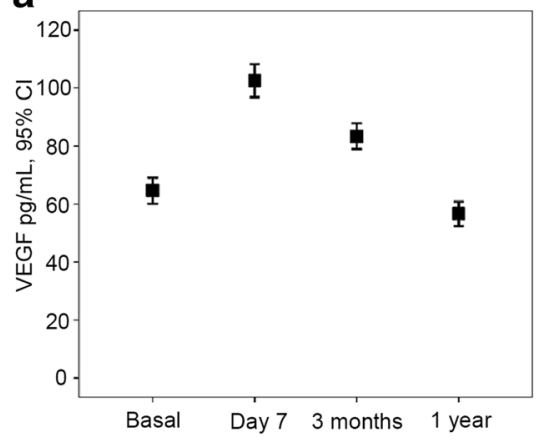

C

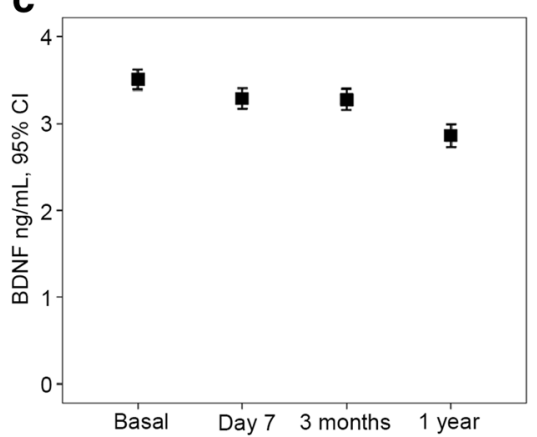

b

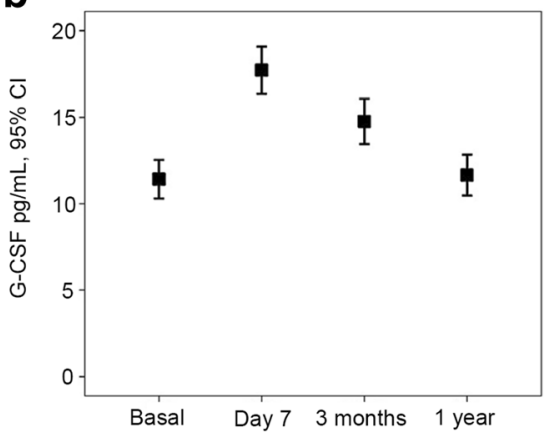

d

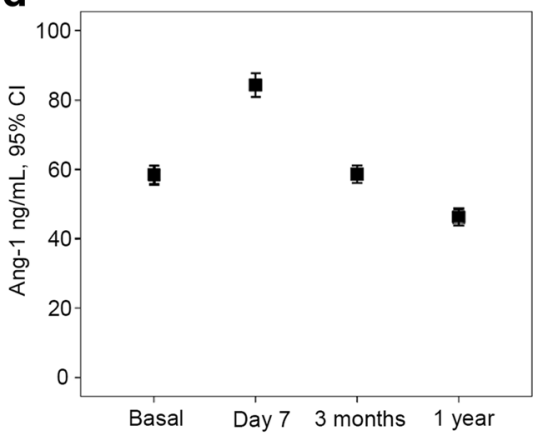

e

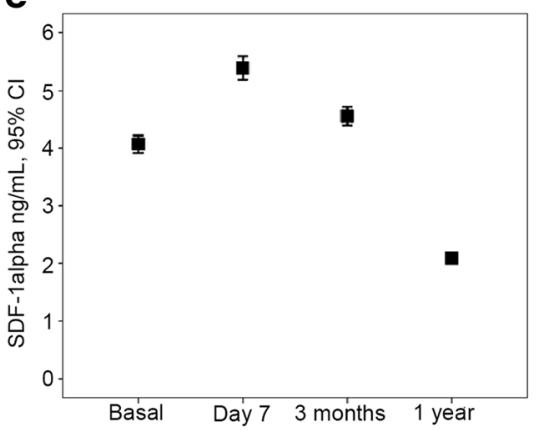


Table 1 Baseline clinical characteristics, vascular risk factors, stroke subtype, biochemical parameters and neuroimaging findings in patients with good or poor outcome at 12 \pm 3 months

\begin{tabular}{|c|c|c|c|}
\hline & Good outcome $n=351$ & Poor outcome $n=201$ & $p$ value \\
\hline Age, years & $66.9 \pm 11.6$ & $69.6 \pm 9.4$ & 0.001 \\
\hline Males, \% & 61.3 & 63.7 & 0.586 \\
\hline History of hypertension, $\%$ & 58.4 & 71.6 & 0.002 \\
\hline History of diabetes, $\%$ & 19.4 & 27.9 & 0.026 \\
\hline Smoking habit, \% & 26.8 & 13.4 & $<0.0001$ \\
\hline Alcohol consumption, $\%$ & 15.4 & 9.5 & 0.051 \\
\hline History of hyperlipidemia, $\%$ & 41.3 & 55.2 & 0.002 \\
\hline History of ischemic heart disease, $\%$ & 15.4 & 16.9 & 0.631 \\
\hline History of atrial fibrillation, $\%$ & 24.8 & 34.8 & 0.014 \\
\hline History of heart failure, $\%$ & 10.3 & 9.5 & 0.883 \\
\hline Previous disability $(\mathrm{mRS}<1$ ) & $0(0,0)$ & $0(0,0)$ & 0.448 \\
\hline Statins prior to stroke, $\%$ & 31.3 & 21.1 & 0.008 \\
\hline Axillary temperature admission, ${ }^{\circ} \mathrm{C}$ & $36.4 \pm 0.5$ & $36.6 \pm 0.5$ & 0.080 \\
\hline Glucose levels, mg/dL & $127.6 \pm 50.4$ & $143.2 \pm 43.5$ & $<0.0001$ \\
\hline Leukocytes, $\times 10^{3} / \mathrm{mmc}$ & $9.3 \pm 6.1$ & $9.1 \pm 2.4$ & 0.100 \\
\hline Fibrinogen, mg/dL & $404.7 \pm 133.4$ & $439.6 \pm 160.1$ & 0.003 \\
\hline NIHSS at admission & $8(5,13)$ & $18(14,20)$ & $<0.0001$ \\
\hline Fibrinolytic treatment, $\%$ & 39.0 & 30.3 & 0.043 \\
\hline Infarct volume (4th to 7 th day), $\mathrm{mL}$ & $16.8 \pm 29.8$ & $98.2 \pm 94.1$ & $<0.0001$ \\
\hline Hemorrhagic transformation, $\%$ & 13.4 & 16.9 & 0.263 \\
\hline Statins after stroke, $\%$ & 70.9 & 62.2 & 0.038 \\
\hline TOAST & & & 0.113 \\
\hline Atherothrombotic, $\%$ & 11.7 & 17.9 & \\
\hline Cardioembolic, $\%$ & 48.1 & 53.2 & \\
\hline Indeterminate, $\%$ & 40.2 & 28.9 & \\
\hline Rehabilitation during-hospital, $\%$ & 27.1 & 33.0 & 0.145 \\
\hline Rehabilitation during 12 months, $\%$ & 10.3 & 18.9 & 0.007 \\
\hline
\end{tabular}

showed good functional outcome at 12 months. Patients with good functional outcome were younger, showed milder stroke severity, and smaller lesion volume at 4 th -7 th day. Furthermore, fibrinolytic treatment, rehabilitation, and statins treatment previous and during acute phase of stroke were more frequent in the group of patients with good outcome.

On the other hand, with the exception of BDNF, all GF and SDF- $1 \alpha$ showed a different expression profile in patients with good and poor outcome. Mean (SD) levels of VEGF, G-CSF, and SDF- $\alpha$ at day 7 and 3 months as well as Ang- 1 at day 7 were significantly higher in patients with good functional outcome (Fig. 3).

In the logistic regression analysis, serum levels of SDF-1 $\alpha$ (OR, 28.5; 95\% CI 13.2-81.2), VEGF (OR, 19.3; 95\% CI 10.7-231.9), Ang-1 (OR, 14.7; 95\% CI 4.4-61.9), and GCSF (OR, 9.6, 95\% CI 6.9-55.3) at day 7 were independently associated with good functional outcome at 12 months after adjustment by significant variables of bivariate analysis showed in Table 1. Moreover, serum levels of SDF-1 $\alpha(\mathrm{OR}$, 20.2; 95\% CI 8.1-57.1), VEGF (OR, 10.9; 95\% CI 2.3-50.7), and G-CSF (OR, 8.9; 95\% CI 4.4-34.7) at 3 months were also independently associated with good functional outcome at 12 months (Table 2).

\section{Secondary Outcome: Good Functional Outcome at 3 Months}

Three hundred and forty-seven patients $(62.9 \%)$ had good functional outcome at 3 months. Bivariate analysis showed similar results to those obtained for the functional prognosis at 12 months (data not shown). The logistic regression analysis showed that serum levels of SDF-1 $\alpha(\mathrm{OR}, 20.6 ; 95 \% \mathrm{CI}$ 9.2-46.1), VEGF (OR, 12.6; 95\% CI 5.7-27.7), Ang-1 (OR, 9.1; 95\% CI 4.0-20.8), and G-CSF (OR, 6.2; 95\% CI 3.221.3 ) at day 7 were independently associated with good functional outcome at 3 months.

\section{Secondary Outcome: Lesion Volume at 4th-7th Days and at 6 Months}

Figures 4 and 5 show the correlations between GF and SDF$1 \alpha$ serum levels, measured at admission and the lesion 

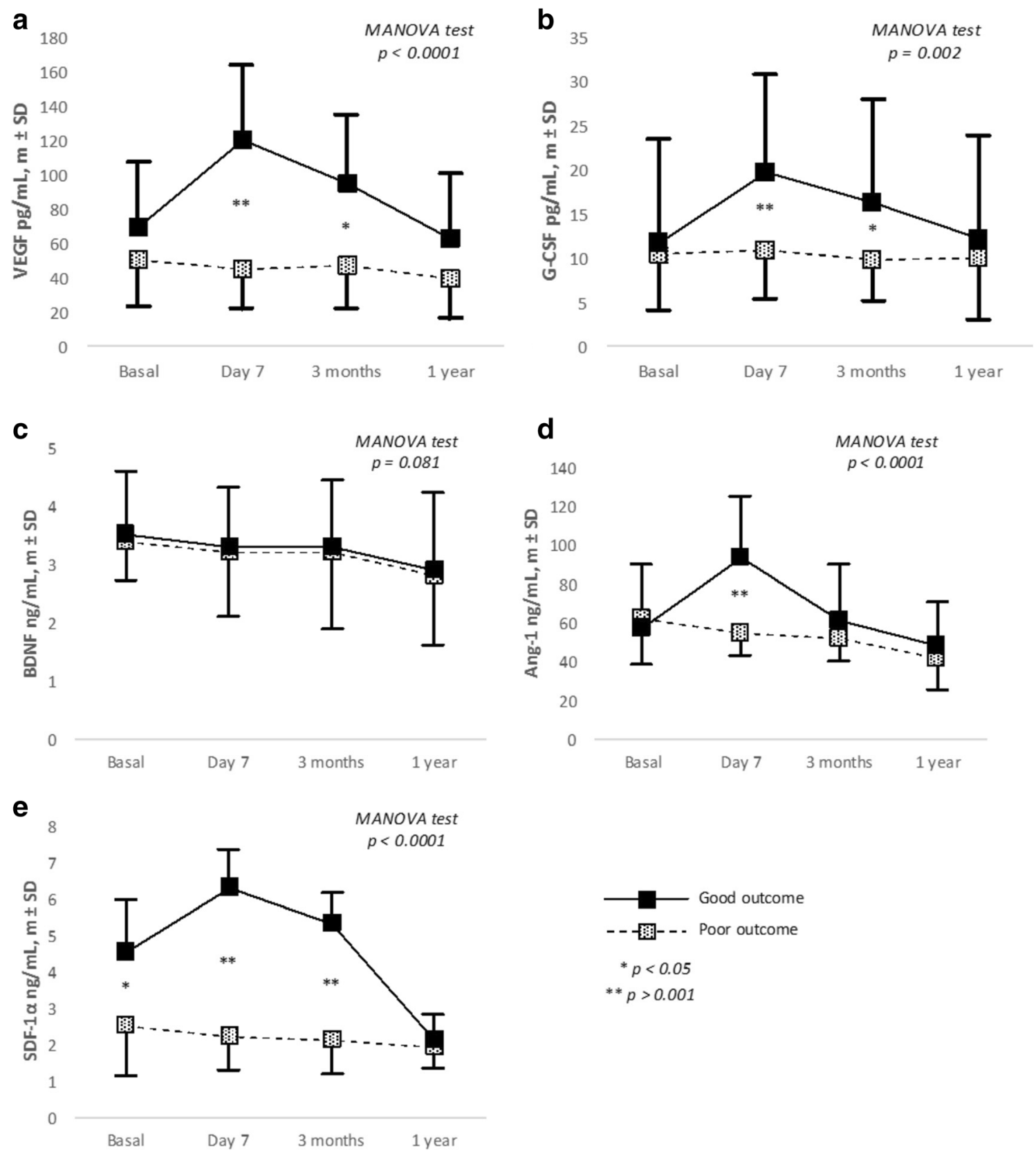

Fig. 3 (a-e) Temporal profile serum levels of GF (VEGF, G-CSF, BDNF, Ang-1) and SDF-1 $\alpha$ in patients with good or poor outcome at $12 \pm 3$ months

volumes at days 4-7, the correlation between GF and SDF- $1 \alpha$ serum levels at day 7 , and lesion volume at 6 months. Only admission serum levels of SDF- $1 \alpha(r=-0.373)$ were associated with lesion volume at 4th-7th day. However, serum levels at day 7 of VEGF ( $r=-0.324)$, G-CSF $(r=-0.140)$, Ang-1 $(r=-0.216)$, and SDF-1 $\alpha(r=-0.580)$, but not BDNF, had a significant correlation with the lesion volume after 3 months.

In the multiple linear regression model, adjusted for those significant variables associated to lesion volume in the univariate analysis (history of arterial hypertension, alcohol consumption, history of atrial fibrillation, fibrinolytic treatment, and NIHSS at admission), serum levels of VEGF (B, -21.4), G-CSF (B, - 14.0), Ang-1 (B, - 13.3), and SDF-1 $\alpha$ (B, 44.6) measured at day 7 were independently associated with lesion volume at 6 months (all $p<0.01$ ). By contrast, only SDF- $1 \alpha$ levels $(B,-29.6)$ at admission were independently associated to lesion volume at 4th-7th day (Table 3).

\section{Discussion}

This prospective and multicenter study shows a relationship between serum GF and SDF- $1 \alpha$ and brain injury in patients 
Table 2 Adjusted and nonadjusted OR of good outcome at 12 months for serum levels of GF (VEGF, G-CSF, BDNF, Ang-1) and SDF- $1 \alpha$ at admission, day $7 \pm$ $1,3 \pm 1$ months, and $12 \pm 3$ months

\begin{tabular}{|c|c|c|c|c|c|c|}
\hline \multirow[t]{2}{*}{ Growth factors } & \multicolumn{3}{|c|}{ Non-adjusted } & \multicolumn{3}{|c|}{ Adjusted* } \\
\hline & OR & $95 \% \mathrm{CI}$ & $p$ & OR & $95 \% \mathrm{CI}$ & $p$ value \\
\hline VEGF at admission & 1.7 & $1.3-2.3$ & 0.001 & 1.7 & $1.5-4.9$ & 0.001 \\
\hline VEGF at 7 days & 26.6 & $14.1-48.7$ & $<0.0001$ & 19.3 & $10.7-231.9$ & $<0.0001$ \\
\hline VEGF at 3 months & 22.3 & $11.7-42.5$ & $<0.0001$ & 10.9 & $2.3-50.7$ & $<0.0001$ \\
\hline VEGF at 12 months & 3.1 & $2.0-4.9$ & $<0.0001$ & 1.6 & $1.1-10.0$ & $<0.0001$ \\
\hline G-CSF at admission & 0.8 & $0.5-1.1$ & 0.255 & 0.85 & $0.4-1.5$ & 0.603 \\
\hline G-CSF at 7 days & 15.9 & $8.8-28.9$ & $<0.0001$ & 9.59 & $6.9-55.3$ & $<0.0001$ \\
\hline G-CSF at 3 months & 6.5 & $3.7-11.4$ & $<0.0001$ & 8.99 & $4.4-34.7$ & $<0.0001$ \\
\hline G-CSF at 12 months & 1.4 & $0.9-2.3$ & 0.124 & 1.11 & $0.5-2.3$ & 0.781 \\
\hline BDNF at admission & 1.3 & $0.7-2.1$ & 0.305 & 0.69 & $0.2-1.9$ & 0.494 \\
\hline BDNF at 7 days & 2.2 & $1.4-3.6$ & 0.001 & 2.69 & $0.9-6.9$ & 0.139 \\
\hline BDNF at 3 months & 1.9 & $1.1-3.1$ & 0.017 & 1.48 & $0.5-3.6$ & 0.395 \\
\hline BDNF at 12 months & 1.3 & $0.7-2.4$ & 0.337 & 1.75 & $0.5-5.1$ & 0.308 \\
\hline Ang-1 at admission & 0.8 & $0.5-1.2$ & 0.279 & 1.47 & $0.7-2.7$ & 0.230 \\
\hline Ang-1 at 7 days & 29.6 & $15.4-57.1$ & $<0.0001$ & 14.72 & $4.4-61.9$ & $<0.0001$ \\
\hline Ang- 1 at 3 months & 1.5 & $0.9-2.2$ & 0.062 & 1.57 & $0.7-3.4$ & 0.251 \\
\hline Ang- 1 at 12 months & 1.3 & $0.8-1.9$ & 0.293 & 1.26 & $0.6-2.6$ & 0.536 \\
\hline SDF- $1 \alpha$ at admission & 22.1 & $10.1-126.8$ & $<0.0001$ & 16.25 & $10.1-87.2$ & $<0.0001$ \\
\hline SDF- $1 \alpha$ at 7 days & 31.9 & $15.8-214.1$ & $<0.0001$ & 28.53 & $13.2-81.1$ & $<0.0001$ \\
\hline SDF- $1 \alpha$ at 3 months & 29.6 & $10.3-103.8$ & $<0.0001$ & 20.19 & $8.1-57.2$ & $<0.0001$ \\
\hline SDF- $1 \alpha$ at 12 months & 2.3 & $1.0-5.3$ & 0.043 & 2.09 & $0.6-7.8$ & 0.269 \\
\hline
\end{tabular}

*Adjusted by age, history of hypertension, history of diabetes, smoking habit, history of hyperlipidemia, history of atrial fibrillation, statins prior to stroke, glucose levels, fibrinogen, NIHSS at admission, fibrinolytic treatment, infarc volume, rehabilitation during 12 months with ischemic stroke. The increase of serum levels of VEGF, Ang-1, G-CSF, and SDF- $1 \alpha$ at day 7 and 3 months after ischemic stroke was independently associated with a good functional outcome at 3 and 12 months. This favorable effect on the primary variable was supported by positive effects on the reduction of lesion volume after 3 months. Importantly, these effects remained significant after adjustment for wellrecognized prognostic factors such as age, lesion volume, stroke severity, and fibrinolytic treatment.

Serum levels of VEGF, G-CSF, Ang- 1 , and SDF- $1 \alpha$ showed a similar biological pattern, with a peak value at day 7 and remained elevated levels during the first 3 months. Of all of them, SDF- $1 \alpha$ was the biomarker more powerful associated to the outcome variables (functional recovery and residual lesion volume). Conversely, BDNF had a different profile, showing a progressive decrease of its serum levels from the stroke onset. In fact, BDNF levels were not associated with any of the endpoints analyzed in this study, neither with de infarct volume, nor with the functional outcome during a follow-up period of 1 year. The role of BDNF in the pathophysiology of ischemic stroke has been evaluated in animal and clinical studies, where the results were not conclusive. Previous studies $[17,18]$ have presented a relationship between a baseline and isolated BDNF determination and outcome of ischemic stroke patients (in our series there was a slight non-significant association with a lower infarct volume); moreover, the increase of the BDNF expression after rehabilitation has also been reported [19]. Other studies supported the lack of correlation between serum BDNF levels and lesion size or recovery in stroke patients [20,21]. However, it is important to note that different research works have showed that serum BDNF may not accurately reflect BDNF concentrations in the brain due to endothelial dysfunction $[22,23]$. BDNF levels could be also influenced by a variety of factors including platelet count, gender, smoking status, depression, and age and Val66Met polymorphism [21, 24-28]. Therefore, further studies are necessary to elucidate the potential role of $\mathrm{BDNF}$ as a GF able to induce recovery in ischemic stroke patients.

The selection of the GF analyzed in this study was performed due to previous studies, mainly in preclinical models of cerebral ischemia, since there is a gap in studies in clinical practice which may be one of the reasons why clinical trials with GF such as G-CSF have failed [13, 14]. Specifically, VEGF is a potent angiogenic factor, inducible by hypoxia, which plays an important role in the vascular response to ischemia, promoting the formation of new cerebral blood vessels (neovascularization) [29-34]. 
Fig. 4 Scatterplots between GF (VEGF, G-CSF, BDNF, Ang-1) and SDF- $1 \alpha$ serum levels measured at admission and $7 \pm 1$ days and the lesion volumes at 4 th -7 th day
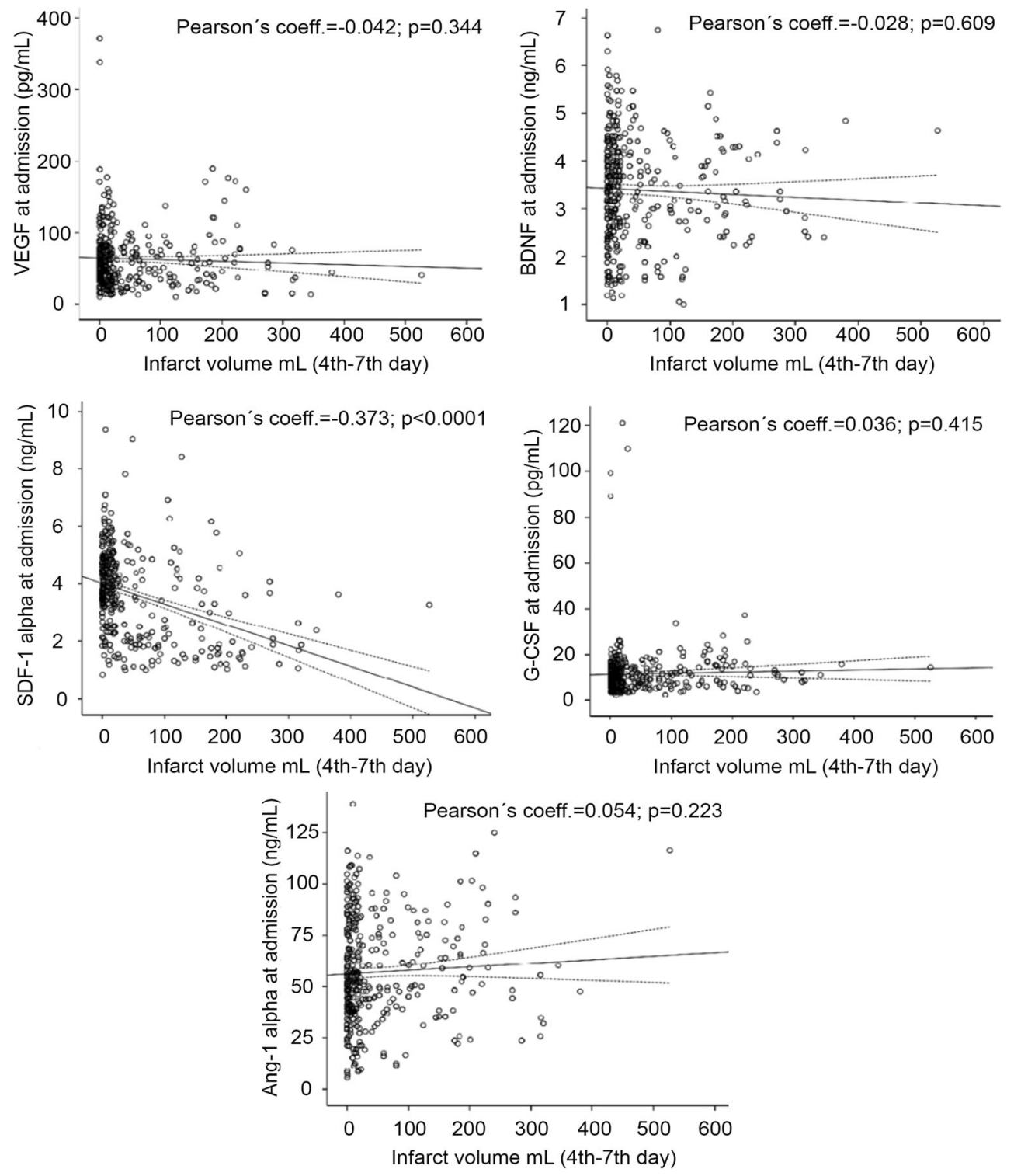

Likewise, VEGF has been also implicated as a factor promoting neurogenesis in the adult brain, increasing the proliferation and differentiation of endothelial and neuronal progenitor cells [9, 29, 35].

G-CSF induces the mobilization of hematopoietic stem cells from the bone marrow to the injured brain regions, reducing the volume of the infarct and improving neuronal plasticity and vascularization, which translates into a better functional recovery [36-38]. Also inhibits apoptosis and stimulates the differentiation of neuronal progenitor cells, inducing neurogenesis.

BDNF is a neurotropic factor involved in ischemiainduced neurogenesis processes, and increased recruitment of endogenous progenitors to injured brain regions, mediating repair mechanisms and neuronal plasticity [39]. BDNF has also been shown to induce synaptogenesis, morphogenesis, and plasticity of dendritic spines, resulting in synapses with functionality [40, 41].

The functionality of GF depends, to a large extent, on the relationship between them and between the cells that induce their expression. Thus, VEGF induces the release of BDNF from endothelial cells [42] and stimulates the formation of pericytes [31]. Similarly, Ang-1 and SDF-1 $\alpha$ could be the link between neurogenesis and angiogenesis, promote neuroblast migration, and post-stroke functional recovery [43, 44].

The usefulness of the knowledge about the potential role of GF in the recovery of the cerebral lesion caused by the ischemia is not conditioned only by a better understanding of the underlying mechanisms that mediate brain repair but also because they may imply more translational therapies than those mediated by cellular therapy. In this regard, drugs that induce the expression of GF are already known, such as $4 \alpha$-phorbol 
Fig. 5 Scatterplots between GF (VEGF, G-CSF, BDNF, Ang-1) and SDF- $1 \alpha$ serum levels measured at $7 \pm 1$ days and after $6 \pm 3$ months
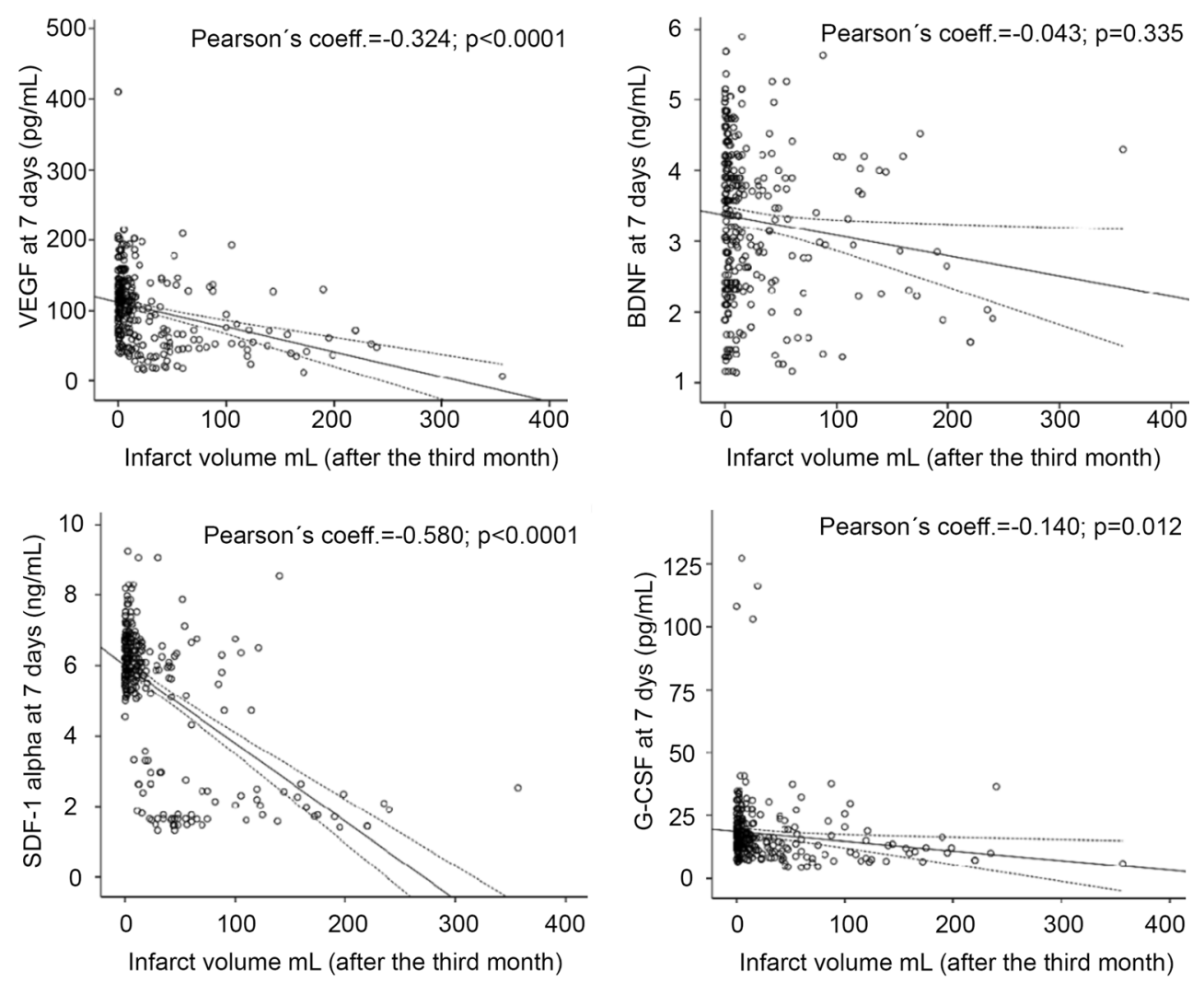

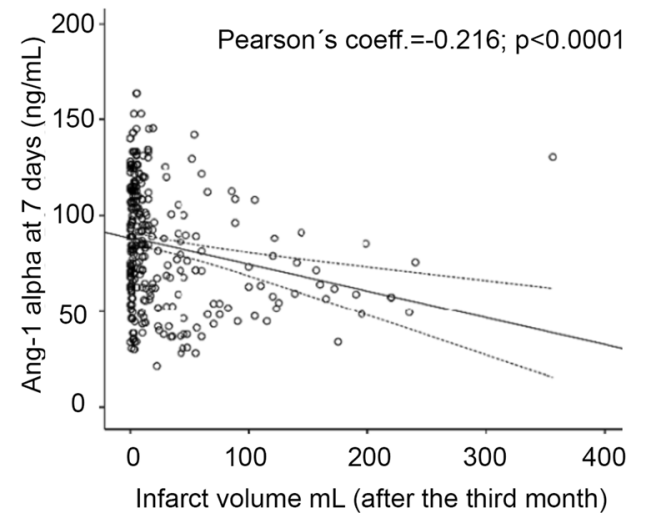

12,13-didecanoate (an endothelial receptor agonist), 15(S)hydroxyeicosat acid (arachidonic acid metabolite) [34], or guanosine [45]. In addition, GF have already been tested in clinical trials, although with modest results. The G-CSF has shown a slight functional improvement, but not of the neurological deficit [14].
Table 3 Adjusted* B of lesion volume at 4 th- -7 th day and after 3 months for serum levels of GF (VEGF, G-CSF, BDNF, Ang-1) and SDF- $1 \alpha$ at admission and day $7 \pm 1$

\begin{tabular}{|c|c|c|c|c|c|c|c|}
\hline \multirow[t]{2}{*}{ Admission } & \multicolumn{3}{|c|}{ Lesion volume (4th-7th day) } & \multirow[t]{2}{*}{ 7th day } & \multicolumn{3}{|c|}{ Infarct volume (at 6 month) } \\
\hline & $B$ & $95 \% \mathrm{CI}$ & $p$ value & & $B$ & $95 \% \mathrm{CI}$ & $p$ value \\
\hline VEGF & -4.9 & -13.6 to 3.79 & 0.269 & VEGF & -21.6 & -29.6 to -3.7 & $<0.0001$ \\
\hline G-CSF & -1.4 & -11.1 to 21.42 & 0.125 & G-CSF & -14.0 & -23.6 to -4.7 & 0.003 \\
\hline BDNF & 3.5 & -11.6 to 18.56 & 0.650 & BDNF & -11.2 & -23.4 to 1.1 & 0.074 \\
\hline Ang-1 & -3.3 & -13.60 to 7.01 & 0.530 & Ang-1 & -13.3 & -24.5 to -2.0 & 0.021 \\
\hline SDF-1 $\alpha$ & -29.2 & -41.95 to -16.50 & $<0.0001$ & SDF- $1 \alpha$ & -44.6 & -54.2 to -34.9 & $<0.0001$ \\
\hline
\end{tabular}

*Adjusted by age, history of atrial fibrillation, NIHSS at admission, and, fibrinolytic treatment 
This study has some limitations. First, patients were admitted to Stroke Units of 6 Spanish hospitals, and hematology and coagulation test were assessed in the central laboratory of each participating hospital. However, patients were treated according to the European Stroke Organisation guidelines [15]. In addition, the growth factors and SDF- $1 \alpha$ determinations of the total patients were performed only in one hospital. Second, to have normal healthy subjects as a control group (cohort of age, comorbidity) would give more value to the results presented. Three, it is important to note that many conditions affect the level of growth factors, for example, subject exercise or age [21, 24-28]. Although given the mean age of patients (68.2 \pm 11.4 years), their physical activity or exercise was low or moderate. Four, we did not take the location of the lesion into account in our analysis, only the lesion volume. Five, we have not considered in the analysis the different reperfusion treatments (intravenous or intraarterial fibrinolysis, thrombectomy, or both procedures). Six, the design of our study does not allow concluding mechanistic aspects on the role of growth factors in the prognosis of patients with cerebral infarction. However, its results open the possibility to design new more translational clinical trials. Short-term administrations of growth factors during the acute or subacute phases of stroke are not expected to modify the prognosis of patients. According to the temporal expression profiles of GF obtained, an administration started within the first week and prolonged during the first 3 months after the stroke seems a more translational approach. However, for this objective, new therapeutic formulations will be necessary [13].

\section{Summary}

High serum levels of VEGF, Ang-1, G-CSF, and SDF- $1 \alpha$ at day 7 and 3 months after ischemic stroke are associated with good functional outcome and smaller residual lesion at 1 year of follow-up. New clinical trials, not focused in acute phase of stroke, should be conducted in order to clarify whether GF may be able to promote functional recovery in ischemic stroke patients.

Funding Information This project was partially supported by grants from the Spanish Ministry of Economy and Competitiveness (SAF2014-56336-R; RTI2018-102165-B-I00 and SAF2017-84267-R), Xunta de Galicia (Consellería Educación: GRC2014/027 and IN607A2018/3), Instituto de Salud Carlos III (PI13/00292, PI14/01879 and PI17/01103), Spanish Research Network on Cerebrovascular Diseases RETICS-INVICTUS PLUS (RD16/0019), and by the European Union FEDER program. T. Sobrino (CP12/03121 \&CPII17/00027) and F. Campos (CP14/00154 \& CPII19/ 00020) are recipients of research contracts from Miguel Servet Program of Instituto de Salud Carlos III. The sponsors did not participate in study design, collection, analysis, or interpretation of the data.

\section{Compliance with Ethical Standards}

Conflict of Interest The authors declare that they have no conflicts of interest.
Ethical Approval and Patient Consent This research was carried out in accordance with the Declaration of Helsinki of the World Medical Association and approved by the Ethics Committee of the participating hospitals. Informed consent was obtained from each patient or their relatives after full explanation of the procedures.

Open Access This article is distributed under the terms of the Creative Commons Attribution 4.0 International License (http:// creativecommons.org/licenses/by/4.0/), which permits unrestricted use, distribution, and reproduction in any medium, provided you give appropriate credit to the original author(s) and the source, provide a link to the Creative Commons license, and indicate if changes were made.

\section{References}

1. Mensah GA, Sacco RL, Vickrey BG, et al. From data to action: neuroepidemiology informs implementation research for global stroke prevention and treatment. Neuroepidemiology. 2015;45: 221-9.

2. Howard G, Goff DC. Population shifts and the future of stroke: forecast of the future burden of stroke. Ann N Y Acad Sci. 2012;1268:14-20.

3. Papanagiotor P, White CJ. Endovascular reperfusion strategies for acute stroke. JACC Cardiovasc Interv. 2016;9:307-17.

4. Calautti C, Baron JC. Functional neuroimaging studies of motor recovery after stroke in adults: a review. Stroke. 2003;34:1553-66.

5. Ramachandran VS. Plasticity and functional recovery in Neurology. Clin Med. 2005;5:368-73.

6. Azad TD, Veeravagu A, Steinberg GK. Neurorestauration after stroke. Neurosurg Focus. 2016;40:E2.

7. Carmichael ST. Emergent properties of neural repair: elemental biology to therapeutic concepts. Ann Neurol. 2016;79:895-906.

8. Bang OY, Kim EH, Cha JM, Moon GJ. Adult stem cell therapy for stroke: challenges and progress. J Stroke. 2016;18:256-66.

9. Sobrino T, Pérez-Montes M, Brea D, Rodríguez-Yáñez M, Blanco M, Castillo J. Temporal profile of molecular signatures associated with circulating endothelial progenitor cells in human ischemic stroke. J Neurosci Res. 2012;90:1788-93.

10. Gutiérrez-Fernández M, Fuentes B, Rodríguez-Frutos B, RamosCejudo J, Vallejo-Cremade MT, Díez-Tejedor E. Trophic factors and cell therapy to stimulate brain repair after ischaemic stroke. J Cell Mol Med. 2012;16:2280-90.

11. Ramos-Cejudo J, Gutiérrez-Fernández M, Otero-Ortega L, et al. Brain-derived neurotrophic factor administration mediated oligodendrocyte differentiation and myelin formation in subcortical ischemic stroke. Stroke. 2015;46:221-8.

12. Peplow PV. Growth factor- and cytokine-stimulated endothelial progenitor cells in post-ischemic cerebral neovascularization. Neural Regen Res. 2014;9:1425-9.

13. Chan SJ, Love C, Spector M, Cool SM, Nurcombe V, Lo EH. Endogenous regeneration: engineering factors for stroke. Neurochem Int. 2017;107:57-65.

14. Huang X, Liu Y, Bai S, Peng L, Zhang B, Lu H. Granulocyte colony stimulating factor therapy for stroke: a pairwise metaanalysis of randomized controlled trial. PLoS ONE. 2017;12: e0175774.

15. European Stroke Organisation (ESO) Executive Committee; ESO Writing Committee. Guidelines for management of ischaemic stroke and transient ischaemic attack 2008. Cerebrovasc Dis. 2008;25:457-507.

16. Adams HP Jr, Bendixen BH, Kappelle LJ, et al. Classification of subtype of acute ischemic stroke. Definitions for use in a 
multicenter clinical trial. TOAST. Trial of Org 10172 in Acute Stroke Treatment. Stroke. 1993;24:35-41.

17. Qiao HJ, Li ZZ, Wang LM, Sun W, Yu JC, Wang B. Association of lower serum brain-derived neurotrophic factor levels with larger infarcts volumes in acute ischemic stroke. J Neuroimmunol. 2017;307:69-73.

18. Zhao H, Alam A, San CY, et al. Molecular mechanisms of brainderived neurotrophic factor in neuro-protection: recent developments. Brain Res. 1665;2017:1-21.

19. Yong MS, Kim SG, Cheon SH. Effects of skilled reach training with affected forelimb and treadmill exercise on the expression of neurotrophic factor following ischemia-induced brain injury in rats. J Phys Ther Sci. 2017;29:647-50.

20. Jiménez I, Sobrino T, Rodríguez-Yáñez M, et al. High serum levels of leptin are associated with post-stroke depression. Psychol Med. 2009;39:1201-9.

21. Yang L, Zhang Z, Sun D, Xu Z, et al. Low serum BDNF may indicate the development of PSD in patients with acute ischemic stroke. Int J Geriatr Psychiatry. 2011;26:495-502.

22. Béjot Y, Mossiat C, Giroud M, Prigent-Tessier A, Marie C. Circulating and brain BDNF levels in stroke rats. Relevance to clinical studies. PLoS One. 2011;6:e29405.

23. Rodier M, Quirié A, Prigent-Tessier A, et al. Relevance of poststroke circulating BDNF levels as a prognostic biomarker of stroke outcome. Impact of rt-PA treatment. PLoS One. 2015;10:e0140668.

24. Bus BA, Molendijk ML, Penninx BJ, et al. Determinants of serum brain-derived neurotrophic factor. Psychoneuroendocrinology. 2011;36:228-39.

25. Fujimura H, Altar CA, Chen R, et al. Brain-derived neurotrophic factor is stored in human platelets and released by agonist stimulation. Thromb Haemost. 2002;87:728-34.

26. Golden E, Emiliano A, Maudsley S, et al. Circulating brain-derived neurotrophic factor and indices of metabolic and cardiovascular health: data from the Baltimore Longitudinal Study of Aging. PLoS One. 2010;5:e10099.

27. Lommatzsch M, Zingler D, Schuhbaeck K, et al. The impact of age, weight and gender on BDNF levels in human platelets and plasma. Neurobiol Aging. 2005;26:115-23.

28. Zhou Z, Lu T, Xu G, Yue X, et al. Decreased serum brain-derived neurotrophic factor (BDNF) is associated with post-stroke depression but not with BDNF gene Val66Met polymorphism. Clin Chem Lab Med. 2011;49:185-9.

29. Sun Y, Jin K, Xie L, et al. VEGF-induced neuroprotection, neurogenesis, and angiogenesis after focal cerebral ischemia. $\mathrm{J}$ Clin Invest. 2003;111:1843-51.

30. Greenberg DA, Jin K. From angiogenesis to neuropathology. Nature. 2005;438:954-9.

31. Jean Le Blanc N, Guruswamy R, El Ali A. Vascular endothelial growth factor isoform-B stimulates neurovascular repair after ischemic stroke by promoting the function of pericytes via vascular endothelial growth factor receptor-1. Mol Neurobiol. 2018;55: 3611-26.

32. Chen CK, Hsu PY, Wang TM, Miao ZF, Lin RT, Juo SH. TRPV4 activation contributes functional recovery from ischemic stroke via angiogenesis and neurogenesis. Mol Neurobiol. 2018;55:4127-35.

33. Xue L, Chen H, Zhang T, Chen J, Geng Z, Zhao Y. Changes in serum vascular endothelial growth factor and endostatin concentrations associated with circulating endothelial progenitor cells after acute ischemic stroke. Metab Brain Dis. 2017;32:641-8.

34. Chen L, Zhu YM, Li YN, et al. The 15-LO-1/15-HETE system promotes angiogenesis by upregulating VEGF in ischemic brains. Neurol Res. 2017;1:1-8.

35. Heissig B, Hattori K, Dias S, et al. Recruitment of stem and progenitor cells from the bone marrow niche requires MMP-9 mediated release of kit-ligand. Cell. 2002;109:625-37.

36. Schneider A, Krüger C, Steigleder T, et al. The hematopoietic factor G-CSF is a neuronal ligand that counteracts programmed cell death and drives neurogenesis. J Clin Invest. 2005;115:2083-98.

37. Shyu WC, Lin SL, Yang HI, et al. Functional recovery of stroke rats induced by granulocyte colony-stimulating factor-stimulated stem cells. Circulation. 2004;110:1847-54.

38. Kawada H, Takizawa S, Takanashi T, et al. Administration of hematopoietic cytokines in the subacute phase after cerebral infarction is effective for functional recovery facilitating proliferation of intrinsic neural stem/progenitor cells and transition of bone marrowderived neuronal cells. Circulation. 2006;113:701-10.

39. Lichtenwalner RJ, Parent JM. Adult neurogenesis and the ischemic forebrain. J Cereb Blood Flow Metab. 2006;26:1-20.

40. Alsina B, Vu T, Cohen-Cory S. Visualizing synapse formation in arborizing optic axons in vivo: dynamics and modulation by BDNF. Nat Neurosci. 2001;4:1093-101.

41. Lessmann V, Gottmann K, Malcangio M. Neurotrophin secretion: current facts and future prospects. Prog Neurobiol. 2003;69:341-74.

42. Louissaint A, Rao S, Leventhal C, Goldman SA. Coordinated interaction of neurogenesis and angiogenesis in the adult songbird brain. Neuron. 2002;34:945-60.

43. Ohab JJ, Fleming S, Blesch A, Carmichael ST. A neurovascular niche for neurogenesis after stroke. J Neurosci. 2006;26:13007-16.

44. Cui X, Chen J, Zacharek A, Roberts C, Yang Y, Chopp M. Nitric oxide donor up-regulation of SDF1/CXCR4 and Ang1/Tie2 promotes neuroblast cell migration after stroke. J Neurosci Res. 2009;87:86-95.

45. Deng G, Qiu Z, Li D, Fang Y, Zhang S. Delayed administration of guanosine improves long-term functional recovery and enhances neurogenesis and angiogenesis in a mouse model of photothrombotic stroke. Mol Med Rep. 2017;15:3999-4004.

Publisher's Note Springer Nature remains neutral with regard to jurisdictional claims in published maps and institutional affiliations. 